\title{
CLIMATE SERVICES FOR INFECTIOUS DISEASE CONTROL: A NEXUS BETWEEN PUBLIC HEALTH PREPAREDNESS AND SUSTAINABLE DEVELOPMENT, LESSONS LEARNED FROM LONG-TERM MULTI-SITE TIME-SERIES ANALYSIS OF DENGUE FEVER IN VIETNAM
}

\author{
Tran Mai Kien'1), Tran Thi Tuyet Hanh2), Tran Khanh Long2), \\ Mikkel B. Quam3), Joacim Rocklov3), Nguyen Huu Quyen'1), \\ Le Thi Thanh Huong2), Hoang Van Minh2)
}

1) Vietnam Institute of Meteorology, Hydrology, and Climate Change, Hanoi, Vietnam; ${ }^{2)}$ Hanoi School of Public Health, Hanoi, Vietnam;

3) Department of Public Health and Clinical Medicine, Epidemiology and Global Health, Umea University, Umea, Sweden; ${ }^{4)}$ Hanoi

Medical University, Hanoi, Vietnam

\begin{abstract}
BACKGROUND: Climate services provide valuable information for making actionable, data-driven decisions to protect public health in a myriad of manners. There is mounting global evidence of the looming threat climate change poses to human health, including the variability and intensity of infectious disease outbreaks in Vietnam and other lowresource and developing areas. In light of the Sustainable Development Goals, this study aimed to examine the utility of spatial and time-series analysis, to inform public health preparedness strategies for sustainable urban development, in terms of dengue epidemiology, surveillance, control, and early warnings.
\end{abstract}

SUBJECTS AND METHODS: Nearly 40 years of spatial and temporal (times-series) dataset of meteorological records, including rainfall, temperature, and humidity (among others) which can be predictors of dengue were assembled for all provinces of Vietnam. This dataset was associated with case data reported to General Department of Preventive Medicine, Ministry of Health of Vietnam, during the same period. Time series of climate and disease variables were analyzed for trend and changing pattern over time. The time-series statistical analysis method sought to identify spatial (when possible) and temporal trend, seasonality, cyclical pattern of disease, and to discover anomalous outbreak events, which departed from expected epidemiological pattern, and corresponding meteorological phenomena, such as El Nino Southern Oscillation (ENSO).

RESULTS: Analysis yielded largely converged findings with other locations in South East Asia for larger outbreak years and events such as ENSO. Seasonality, trend, and cycle in many provinces were persistent throughout the dataset, indicating strong potential for climate services to be used in dengue early warnings.

CONCLUSION: Public health practitioners, having adequate tools for dengue control available, must plan and budget vector control and patient treatment efforts well in advance of large scale dengue epidemics to curb such events with overall morbidity and mortality. Urban and sustainable 
development in Vietnam might benefit from evidence linking climate change and ill-health events spatially and temporally in future planning. Long term analysis of dengue case data and meteorological records, provided a cases study evidence for emerging opportunities that on how refined climate services, could contribute to protection of public health.

Keywords: dengue, Vietnam, climate change, time-series analysis, climate service 